\title{
Calculation of shear viscosity using Green-Kubo relations within a parton cascade
}

\author{
C. Wesp, ${ }^{1}$ A. El,${ }^{1}$ F. Reining, ${ }^{1}$ Z. Xu,${ }^{1,2}$ I. Bouras,${ }^{1}$ and C. Greiner ${ }^{1}$ \\ ${ }^{1}$ Institut für Theoretische Physik, Johann Wolfgang Goethe-Universität, \\ Max-von-Laue-Str. 1, D-60438 Frankfurt am Main, Germany \\ ${ }^{2}$ Frankfurt Institute for Advanced Studies, \\ Ruth-Moufang-Str. 1, D-60438 Frankfurt am Main, Germany
}

(Dated: June 30, 2011)

\begin{abstract}
The shear viscosity of a gluon gas is calculated using the Green-Kubo relation. Time correlations of the energy-momentum tensor in thermal equilibrium are extracted from microscopic simulations using a parton cascade solving various Boltzmann collision processes. We find that the pQCD based gluon bremsstrahlung described by Gunion-Bertsch processes significantly lowers the shear viscosity by a factor of $3-8$ compared to elastic scatterings. The shear viscosity scales with the coupling as $\eta \sim 1 /\left(\alpha_{s}^{2} \log \left(1 / \alpha_{s}\right)\right)$. For constant $\alpha_{s}$ the shear viscosity to entropy density ratio $\eta / s$ has no dependence on temperature. Replacing the pQCD-based collision angle distribution of binary scatterings by an isotropic form decreases the shear viscosity by a factor of 3 .
\end{abstract}




\section{INTRODUCTION}

Results from the Relativistic Heavy Ion Collider (RHIC) at Brookhaven and the Large Hadron Collider (LHC) at CERN suggest the formation of a new state of matter, the quark gluon plasma (QGP) in ultra-relativistic heavy ion collisions. Large values of the elliptic flow $v_{2}$ observed in these experiments [1 7 ] lead to the indication that the QGP behaves like a nearly perfect fluid, i.e. its shear viscosity to entropy density ratio is small. This makes dissipative hydrodynamics a promising candidate to describe the collective behavior of QGP [8 13]. However, hydrodynamics only works if a system is close to thermal equilibrium, which is not applicable for the state of matter shortly after the initial heavy-ion collision where transport theory based models are a more suitable approach [14 17]. One of today's challenge in theoretical nuclear physics is to find a transition between the transport and hydrodynamic description as well as an appropriate set of transport parameters, for example the shear viscosity $\eta$ of the collective medium. In [18] a full leading order pQCD evaluation of shear viscosity in the collinear approximation of gluon splitting is reported. Calculations with AdS/CFT correspondence in $\mathcal{N}=4$ super-symmetric Yang-Mills theory in large $N$ and strong coupling limit show a small universal but non-vanishing lower bound of $\eta / s=1 / 4 \pi$ [19]. A recent analysis within a quasi-particle description and employing a Boltzmann-Vlasov ansatz in the relaxation time approximation also gives a small number when extrapolating in the non perturbative regime[20]. Considering the fully microscopic transport description BAMPS (Boltzmann Approach of Multi-Parton Scattering) while employing binary elastic $g g \rightarrow g g$ and also inelastic bremsstrahlung $g g \leftrightarrow g g g$ pQCD collisions, the shear viscosity was extracted by using approaches motivated from first and second order hydrodynamics [21, 22]. A small viscosity to entropy ratio close to the Ads/CFT conjecture resulted, which is due to the incorporation of the bremsstrahlung processes being more efficient because of larger momentum deflection. Similar ideas have been given in [23, 24] by also using pQCD elastic and inelastic matrix elements and modeling the equilibrium deviations of the distribution function in consistency with kinetic theory. Most recently, in [25] the shear viscosity is extracted also from a microscopic transport description employing using the Green-Kubo relation and perturbative QCD matrix elements for elastic scattering.

In this work we will extract the shear viscosity of a gluon gas from microscopic transport calculations with BAMPS employing also directly the Green-Kubo relation. The Green- 
Kubo relation does not rely on model assumptions or the form of equilibrium deviations. Thus this work can provide an independent cross check to the previously published works [21, 22]. In principle, the present calculations are rather close to those of [25]. In Sec. II] we give a brief overview of the method. In Sec. III the parton cascade BAMPS and numerical setups are briefly introduced. We demonstrate in Sec. IV how to extract the shear viscosity numerically. The results are presented in Sec. V and are compared to previously published calculations. The dependence of the shear viscosity on the pQCD coupling constant $\alpha_{s}$, temperature, and the distribution of the collision angle are investigated and discussed. A comparison with recent results presented in [25] are be discussed in Sec. VC

\section{GREEN-KUBO RELATIONS}

Green and Kubo showed in serial papers [26, 27] that transport coefficients like heat conductivity, shear- and bulk viscosity can be related to the correlation functions of the corresponding flux or tensor in thermal equilibrium. The physical motivation is given by Onsager's regression hypothesis [28], stating that fluctuations are present in every equilibrated system. Dissipation of fluctuations has the same origin as the relaxation towards equilibrium once the system is disturbed by an external force. Both the dissipation and the relaxation time scales are determined by the same transport coefficients.

In this paper we concentrate on shear viscosity $\eta$. The corresponding Green-Kubo relation has the following form [29]:

$$
\eta=\frac{1}{10 \mathrm{~T}} \int_{0}^{+\infty} \mathrm{d} t \int_{V} \mathrm{~d}^{3} r\left\langle\pi^{i j}(\mathbf{r}, t) \pi^{i j}(0,0)\right\rangle,
$$

where $T$ is the temperature, $\pi^{i j}, i, j=x, y, z$ are components of the shear stress tensor, and $\langle\cdots\rangle$ denotes the ensemble average in thermal equilibrium. The correlation between shear components at time $t=0$ and at $t$ is the sum over $i$ and $j$. Furthermore one can show that $\left\langle\pi^{i j}(\mathbf{r}, t) \pi^{i j}(0,0)\right\rangle=10\left\langle\pi^{x y}(\mathbf{r}, t) \pi^{x y}(0,0)\right\rangle$.

The Green-Kubo relation (1) is the long wave limit $k, \omega \rightarrow 0$ of the Fourier transform of the integrated correlation function [29]. Its derivation can be found elsewhere [30 32].

Within kinetic theory, the correlation of shear components can be computed by employing transport simulations for a particle system embedded in a static volume. Simulations using the hadron cascade model UrQMD [33, 34] and the parton cascade model PCM [35] have 
been performed to calculate the shear viscosity of a hadron gas [36] and of a quark-gluon plasma [25].

In this paper we use the parton cascade BAMPS [14, 37] to calculate the shear viscosity of a gluon gas including perturbation QCD based bremsstrahlung processes $g g \leftrightarrow g g g$.

\section{THE PARTON CASCADE BAMPS AND NUMERICAL SETUPS}

The parton cascade BAMPS solves the ultrarelativistic Boltzmann equation

$$
p^{\mu} \partial_{\mu} f(x, p)=C(x, p)
$$

for on-shell particles. Their interactions are simulated via Monte Carlo techniques based on the stochastic interpretation of transition rates [14]. Collision probabilities of two particles in a spatial cell of a volume of $V_{\text {cell }}$ and within a time step $\Delta t$ are

$$
P_{22,23}=v_{\text {rel }} \frac{\sigma_{22,23}}{N_{\text {test }}} \frac{\Delta t}{V_{\text {cell }}},
$$

where $\sigma_{22,23}$ are the total cross section for a $2 \rightarrow 2$ and a $2 \rightarrow 3$ collision, respectively. $v_{\text {rel }}=\left(p_{1}+p_{2}\right)^{2} /\left(2 E_{1} E_{2}\right)$ denotes the relative velocity of the two incoming particles with four momenta $p_{1}, p_{2}$. The probability for the back reaction $3 \rightarrow 2$ is accordingly

$$
P_{32}=\frac{1}{8 E_{1} E_{2} E_{3}} \frac{I_{32}}{N_{\text {test }}^{2}} \frac{\Delta t}{V_{\text {cell }}^{2}},
$$

where $I_{32}$ is a quantity corresponding to a cross section for $3 \rightarrow 2$ processes and is given by integrating over the final states in an interaction process [14].

Between the collisions the particles propagate via free-streaming. Simulations of the space-time evolution of the particles are performed in a static box with periodic boundary conditions.

In order to ensure an accurate solution of the Boltzmann equation, the volume of spatial cells has to be chosen as small as possible, which obviously enhances statistical fluctuations in the simulation. To reduce these fluctuations a test particle method [14] is introduced: the particle number is artificially increased by a factor of $N_{\text {test }}$. Thus, the collision probability has to be reduced by the same factor to keep the particle mean-free path independent of the value of $N_{\text {test }}$. 
For the present simulations, the length of cubic cells is set to be a factor of 3 smaller than the mean free path of particles, and the box length is a factor of 8 larger than the cell length. $N_{\text {test }}$ is chosen to have on average 12 test particles in each cell.

However, the ratio of mean free path to box length does not influence the volume averaged correlation function as long as particles itself stay uncorrelated, which is the case when the total number of particles is large enough [38].

\section{EXTRACTION OF SHEAR VISCOSITY}

We calculate $\left\langle\pi^{x y}(\mathbf{r}, t) \pi^{x y}(0,0)\right\rangle$ numerically according to the Green-Kubo relation (1). The shear component $\pi^{x y}$ is defined as

$$
\pi^{x y}(\mathbf{r}, t)=T^{x y}(\mathbf{r}, t)=\int \frac{\mathrm{d}^{3} \mathbf{p}}{(2 \pi)^{3} E} p^{x} p^{y} f(\mathbf{r}, t ; \mathbf{p})
$$

In the numerical simulation the volume averaged shear tensor is used

$$
\bar{\pi}^{x y}(t)=\frac{1}{V} \sum_{i=1}^{N} \frac{p_{i}^{x} p_{i}^{y}}{E_{i}}
$$

where the sum is over all particles in the box at time $t . V$ is the volume of the box. The correlation of $\bar{\pi}^{x y}$ is obtained by time and ensemble average in the limit $T_{t} \rightarrow \infty$

$$
\left\langle\bar{\pi}^{x y}(t) \bar{\pi}^{x y}(0)\right\rangle=\left\langle\frac{1}{T_{t}} \int_{0}^{T_{t}} \bar{\pi}^{x y}\left(t+t^{\prime}\right) \bar{\pi}^{x y}\left(t^{\prime}\right) \mathrm{d} t^{\prime}\right\rangle=\left\langle\frac{1}{N_{t}} \sum_{j=0}^{N_{t}-1} \bar{\pi}^{x y}(i \Delta t+j \Delta t) \bar{\pi}^{x y}(j \Delta t)\right\rangle,
$$

where $N_{t}=T_{t} / \Delta t$ and $i=t / \Delta t$.

With (6) and (7) the receive the following Green-Kubo relation:

$$
\eta=\frac{V}{10 T} \int_{0}^{\infty} \mathrm{d} t\left\langle\bar{\pi}^{x y}(t) \bar{\pi}^{x y}(0)\right\rangle
$$

Figure 1 shows the fluctuation of $\pi^{x y}$ in one event (left panel) and its correlations over time and ensemble average (right panel) calculated using BAMPS. Results are obtained for elastic binary collisions assuming constant cross sections with isotropic distribution of the collision angle. The initial distribution is the Boltzmann distribution at a temperature of $T=400 \mathrm{MeV}$

$$
f(\mathbf{p})=d_{G} e^{-E / T}
$$



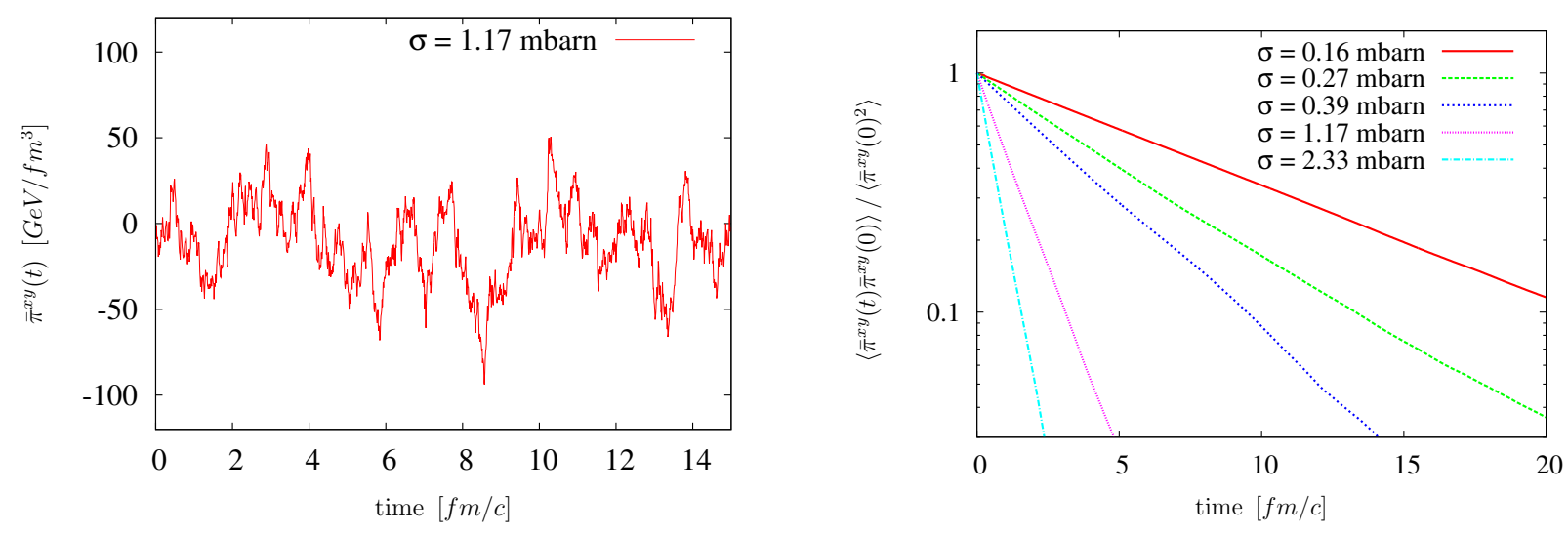

FIG. 1: (Color online) Left: Example for the equilibrium fluctuation of the shear component $\pi^{x y}$. Right: Correlations for different isotropic and constant cross sections. Results are normalised by $\left\langle\left(\bar{\pi}^{x y}\right)^{2}\right\rangle$.

with the degeneracy factor $d_{G}=16$ for of gluons. We neglect Bose enhancement, which is a quantum effect of gluons and has not been implemented in BAMPS yet.

From Fig. 1 we see that the correlation function is an exponentially decreasing function as expected for solutions of the Boltzmann equation with stochastic interpreted cross-sections [39],

$$
\left\langle\bar{\pi}^{x y}(t) \bar{\pi}^{x y}(0)\right\rangle=\left\langle\bar{\pi}^{x y}(0) \bar{\pi}^{x y}(0)\right\rangle e^{-t / \tau},
$$

with a relaxation time scale $\tau$. The initial variance of $\bar{\pi}^{x y}$ can be calculated analytically and is given by

$$
\left\langle\bar{\pi}^{x y}(0) \bar{\pi}^{x y}(0)\right\rangle=\frac{4}{15} \frac{e T}{V},
$$

where $e=3 d_{G} T^{4} / \pi^{2}$ is the gluon energy density. Inserting Eqs. (10) and (11) into Eq. (1) we obtain

$$
\eta=\frac{4}{15} e \tau
$$

Equation (12) is exactly the same as derived in Refs. [21, 40], if one interprets the relaxation time as the inverse of the transport collision rate. In general, the relaxation time scale $\tau$ depends on temperature and individual matrix elements of interactions. Its relationship with microscopic scales will be shown later explicitly. The numerical task is now to find $\tau$ by an appropriate fit to the numerically calculated correlation function. 


\section{NUMERICAL RESULTS}

In this section we present results of shear viscosity. At first we assume constant cross sections for binary elastic collisions with isotropic distribution of the collision angle. In this case we are able to cross-check our numerical results with analytical calculations. Then we calculate the shear viscosity of gluons including pQCD-based elastic and bremsstrahlung processes.

\section{A. Isotropic cross sections}

For binary elastic collisions with isotropic distribution of the collision angle the shear viscosity of an ultrarelativistic Maxwell-Botzmann gas is known from the derivation in the Navier-Stokes approximation [41, 42], which is

$$
\eta^{N S}=1.2654 \frac{T}{\sigma_{22}}
$$

where $\sigma_{22}$ is the total cross section for binary elastic collisions. Comparing Eq. (13) to Eq. (12) leads to $\tau=1.58 /\left(n \sigma_{22}\right)=1.58 \lambda_{m f p}$, i.e., in this case the relaxation time is 1.58 times the mean free path.

Figure 2 shows our results in open circles, compared with Eq. (13) represented by a solid line. The standard deviations are small and displayed within circle areas. We see an excellent agreement between our numerical results and the analytical ones, which proves the applicability of our numerical method within BAMPS.

We also calculate the shear viscosity for inelastic $2 \leftrightarrow 3$ process with a constant cross section $\sigma_{23}$ and isotropic distributions of the collision angles. In this case we have [14]

$$
I_{32}=\frac{192}{d_{G}} \pi^{2} \sigma_{23}
$$

for computing the interaction probability $P_{32}$ for the $3 \rightarrow 2$ process.

In Fig. 2 the results of the shear viscosity for such inelastic processes, $\eta_{23}$, are given by the open squares. Compared with the shear viscosity for binary elastic collisions, $\eta_{22}$, we find that $\eta_{23}$ is a factor of 1.5 smaller than $\eta_{22}$ for $\sigma_{22}=\sigma_{23}$, as indicated by the dashed line. This finding agrees with previously reported numerical [43] and recent analytical calculation 44. 


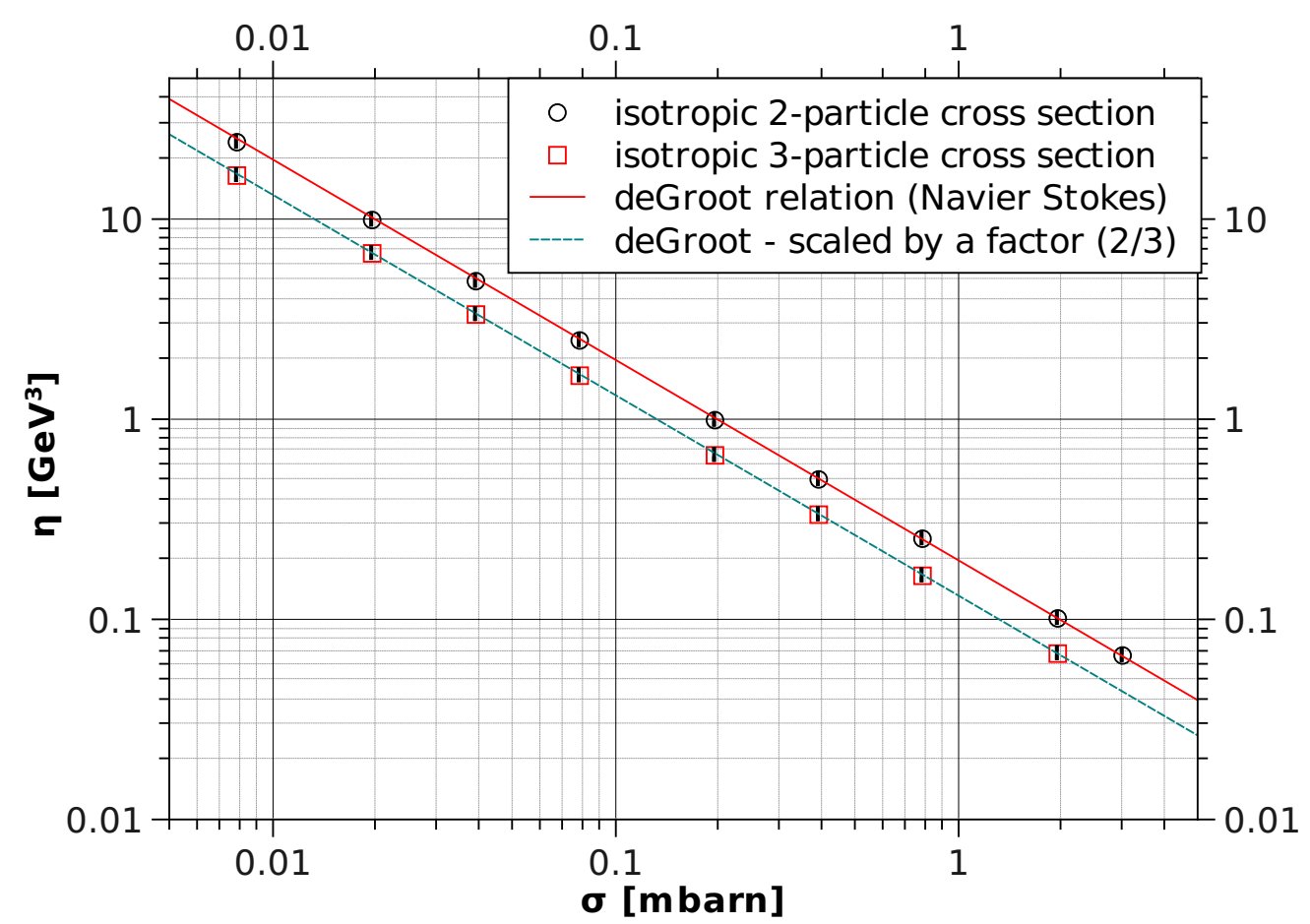

FIG. 2: (Color online) Shear viscosity for a gas with constant and isotropic cross sections. The open circles (squares) are results for elastic $2 \rightarrow 2$ (inelastic $2 \leftrightarrow 3$ ) process. Temperature is fixed at $T=400 \mathrm{MeV}$.

\section{B. pQCD based cross sections}

We now consider elastic and bremsstrahlung processes of gluons based on perturbation QCD (pQCD). The cross sections and matrix elements are the same as used in previous studies [14, 21, 22].

For elastic interactions of gluons, $g g \leftrightarrow g g$, we use the Debye screened cross section in small angle approximation

$$
\frac{d \sigma_{g g \rightarrow g g}}{d q_{\perp}^{2}} \approx \frac{9 \pi \alpha_{s}^{2}}{2} \frac{1}{\left(q_{\perp}^{2}+m_{D}^{2}\right)^{2}} .
$$

The Debye screening mass is computed dynamically from the local particle distribution $f=f(p, x, t)$ via

$$
m_{D}^{2}=d_{G} \pi \alpha_{s} \int \frac{\mathrm{d}^{3} p}{(2 \pi)^{2}} \frac{1}{p} N_{c} f
$$

where $d_{G}=16$ is the gluon degeneracy factor for $N_{c}=3$. 
Inelastic $g g \leftrightarrow g g g$ processes are treated via an effective matrix element based on the work by Gunion and Bertsch [45]. Detailed balance between gluon multiplication and annihilation processes is ensured by the relation $\left|\mathcal{M}_{g g \rightarrow g g g}\right|^{2}=d_{G}\left|\mathcal{M}_{g g g \rightarrow g g}\right|^{2}$. For the case of bremsstrahlung-like processes the matrix element employed in BAMPS reads

$$
\left|\mathcal{M}_{g g \rightarrow g g g}\right|^{2}=\frac{72 \pi^{2} \alpha_{s}^{2} s^{2}}{\left(\mathbf{q}_{\perp}^{2}+m_{D}^{2}\right)^{2}} \frac{48 \pi \alpha_{s} \mathbf{q}_{\perp}^{2}}{\mathbf{k}_{\perp}^{2}\left[\left(\mathbf{k}_{\perp}-\mathbf{q}_{\perp}\right)^{2}+m_{D}^{2}\right]} \Theta\left(\Lambda_{g}-\tau\right)
$$

$\mathbf{q}_{\perp}$ and $\mathbf{k}_{\perp}$ denote the perpendicular components of the momentum transfer and of the radiated gluon momentum in the center of momentum (CM) frame of the colliding particles, respectively.

When considering bremsstrahlung processes the LPM-effect [46], a coherence effect named after Landau, Pomeranchuk and Migdal, needs to be taken into account that leads to a suppression of the emission rate for low $p_{\perp}$ particles. Since such an interference effect cannot be incorporated directly into a semiclassical microscopic transport model such as BAMPS, we choose an effective approach by introducing the Theta function in (17). This implies that the formation time of the emitted gluon must not exceed the mean free path of the parent gluon $g$, ensuring that successive radiative processes are independent of each other.

We calculate the shear viscosity for a temperature of $T=400 \mathrm{MeV}$. Taking the coupling $\alpha_{s}$ as constant, the shear viscosity to the entropy density ratio $\eta / s$ will not depend on temperature for the considered gluon interactions in our case [21] (see later in Sec. VC). From next-to-leading-log calculation the shear viscosity should roughly scale with $\eta_{N N L} \sim$ $g^{-4} T^{3}$ (though it has an additional logarithmic dependence on $T$ due to Debye screening mass) [18] while the entropy of a quark gluon plasma should scale in leading order with $s \sim$ $T^{3}$ [47], which implies a very weak temperature dependence for $\mathrm{T}=300 \mathrm{MeV} \ldots 600 \mathrm{MeV}$

Figure 3 and Table I present the $\eta / s$ ratio of a gluon gas with the pQCD-based interactions, which are the main results of this paper. Because gluons are considered as Boltzmann particles, we have $s=4 n=4 d_{G} T^{3} / \pi^{2}$.

For pure gluon elastic interactions the fit function (dashed curve) agrees with the analytical result of [48, 49] at small $\alpha_{s}$. Other numerical results from [20] confirm this $\left(\alpha_{s}^{2} \log \left(1 / \alpha_{s}\right)\right)^{-1}$ scaling. However, in [50 52] a different scaling $\eta \sim\left(\alpha_{s} \log \left(1 / \alpha_{s}\right)\right)^{-1}$ was suggested, which is a clear difference to the above findings. This different behavior stems from the ansatz that the collision width $\tau^{-1} \sim \alpha_{s} T \log \left(1 / \alpha_{s}\right)$ is employed in their treatment 


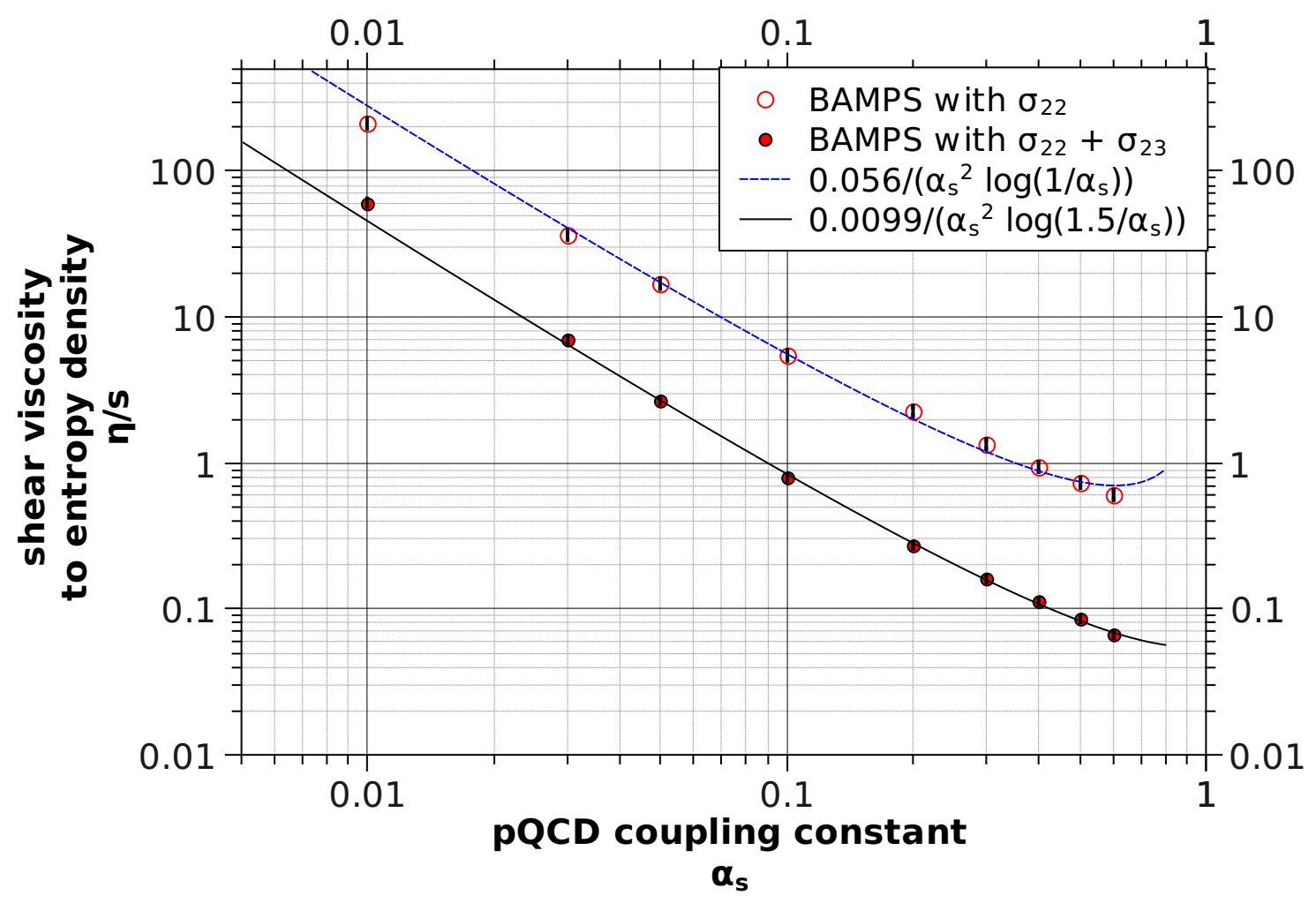

FIG. 3: (Color online) Shear viscosity to entropy density ratio for a gluon gas with $\mathrm{pQCD}$-based interactions.

of a simplified Boltzmann collision process in the relaxation time approximation.

If the gluon bremsstrahlung $g g \leftrightarrow g g g$ is included, the shear viscosity decreases by a factor of 7-8 for $\alpha_{s}>0.1$ and a factor of $3-6$ for $\alpha_{s}<0.1$. The ratio of $\eta_{22} / \eta_{22+23}$ is much larger than that of [18]. The reason was already pointed out in [21]. While in the $\alpha_{s} \rightarrow 0$ limit the collinear elastic processes are clearly dominant, for non-vanishing $\alpha_{s}$ the gluon radiation prefers rather large angles in the Bethe-Heitler regime [53]. In gluon radiation processes the distribution of the radiation angle is close to isotropic [37, 53]. For the $\eta / s$ ratio implementing both elastic and inelastic processes the fit function is found to be $0.0099\left(\alpha_{s}^{2} \log \left(1.5 / \alpha_{s}\right)\right)^{-1}$ and is shown by the solid curve in Fig. 3. This scaling holds for rather small values of $\alpha_{s}$. In the limit of $\alpha_{s} \rightarrow 0$ where bremsstrahlung becomes nearly collinear a scaling $\left(\alpha_{s}^{3} \log \left(1 / \alpha_{s}\right)\right)^{-1}$ is expected for the inelastic contributions [21].

Figure 4 shows comparisons of the present results with those previously reported by some of the authors. The dotted curve is taken from [21] and is obtained by using a gradient expansion in the linearized Boltzmann equation. The deviation from thermal equilibrium 


\begin{tabular}{ccc}
\hline \hline$\alpha_{s}$ & $\frac{\eta}{s}(g g \leftrightarrow g g)$ & $\frac{\eta}{s}(g g \leftrightarrow g g \& g g \leftrightarrow g g g)$ \\
\hline 0.01 & $215 \pm 19$ & $60 \pm 5$ \\
0.03 & $36.4 \pm 1.5$ & $6.9 \pm 0.3$ \\
0.05 & $17.04 \pm 0.73$ & $2.7 \pm 0.1$ \\
0.1 & $5.51 \pm 0.17$ & $0.795 \pm 0.025$ \\
0.2 & $2.21 \pm 0.07$ & $0.28 \pm 0.04$ \\
0.3 & $1.342 \pm 0.035$ & $0.166 \pm 0.025$ \\
0.4 & $0.952 \pm 0.03$ & $0.114 \pm 0.004$ \\
0.5 & $0.74 \pm 0.02$ & $0.087 \pm 0.004$ \\
0.6 & $0.61 \pm 0.02$ & $0.0664 \pm 0.002$ \\
\hline
\end{tabular}

TABLE I: Data plotted in Fig. 3 .

$\phi$, which is needed to calculate the shear viscosity, is approximated by $\phi \sim(\chi / T)\left(p_{z}^{2} / E\right)$, where $\chi$ is a constant. Actually, $\chi$ could be a function of momentum as indicated in the Grad's ansatz, $\phi \sim C_{0} \pi_{\mu \nu} p^{\mu} p^{\nu}$, where $C_{0}$ is a function of temperature. It seems that the simple choice of $\phi$ in [21] makes the $\eta / s$ values lower than the present results at small $\alpha_{s}$. From Fig. 4 we see that the present results have a perfect agreement with those from [22] (dashed curve). In [22] the shear viscosity is derived by identifying the entropy production in hydrodynamic (Israel-Stewart) and kinetic (Boltzmann) approach. For the deviation from thermal equilibrium the Grad's ansatz is used in [22]. In Fig. 4 we also show new results (star symbols) from another work done by Reining et al. [54]. There the shear viscosity is calculated as a ratio of the shear pressure to the velocity gradient in a stationary shear flow pattern. These new results agree well with the present ones. In summary, as noted earlier in [21], pQCD inelastic interactions can drive the gluon matter to a strongly coupled system with an $\eta / s$ ratio as small as the lower bound from the AdS-CFT conjecture.

\section{Temperature and collision angle dependence of the shear viscosity}

In this subsection we study the dependence of the shear viscosity on temperature and the distribution of the collision angle. We only consider elastic gluon collisions in order to repeat the calculations performed in [25]. Our results are shown in Fig. 5 and show some 


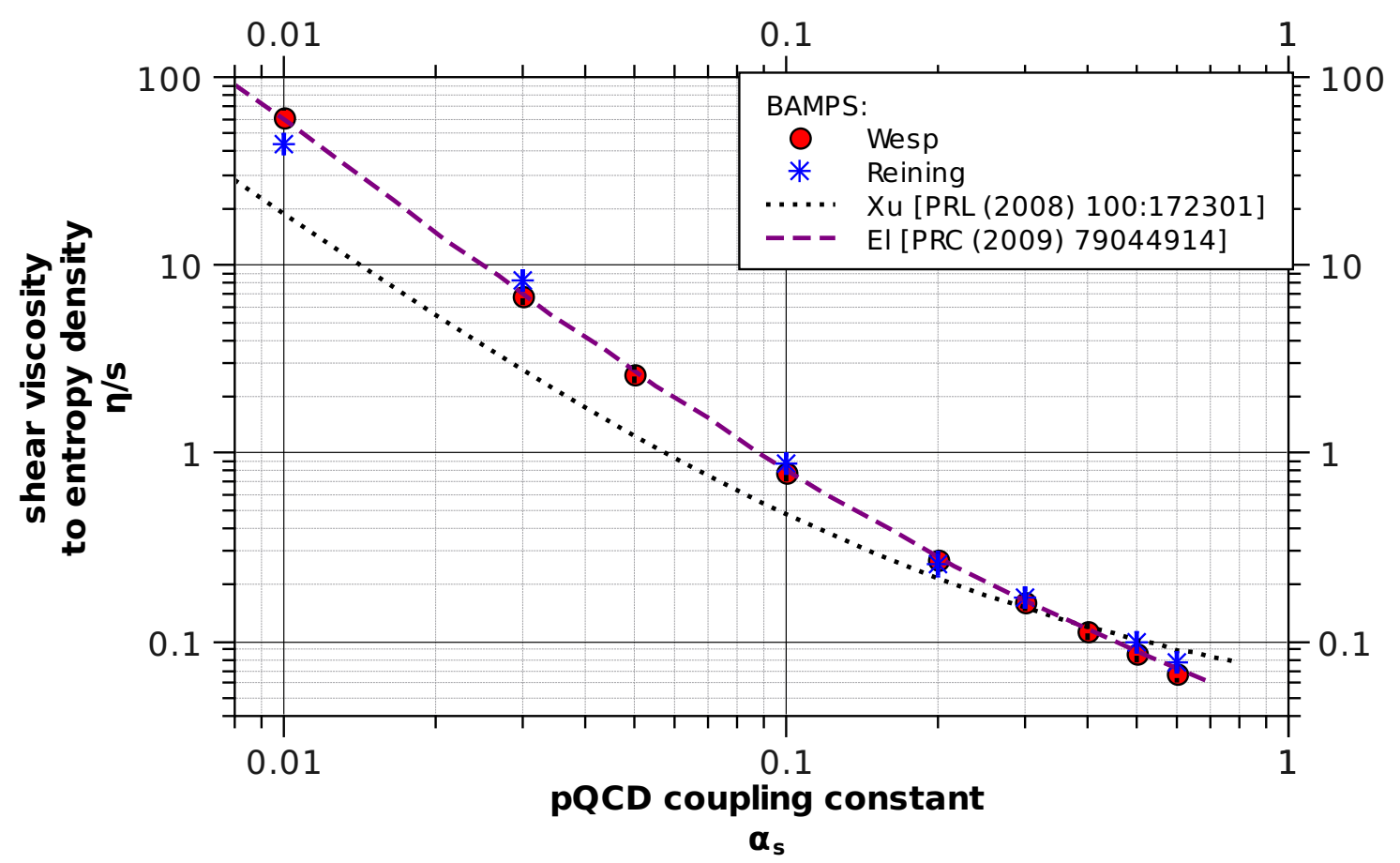

FIG. 4: (Color online) Comparisons of the results in Fig. 3 with those of Refs. [21, 22, 54].

discrepancies with those of [25] at lower temperatures. At temperatures above $400 \mathrm{MeV}$ our results are rather close to theirs.

As already done in the calculations presented in the previous subsection, the cross section of elastic gluon interactions is taken in the small-angle approximation (15). The $\eta / s$ ratios for the elastic gluon interactions at $\alpha_{s}=0.3$ and at three chosen temperatures are shown in Fig. 5 by the circles. They agree well with a constant indicated by the line. There is no temperature dependence of the shear viscosity to entropy ration $\eta / s$ for the considered interactions, which is indicated in the findings of [25]. The authors of [25] employed the same cross section Eq. (15) and calculated the $\eta / s$ ratio according to the Green-Kubo relation (1) by using a transport model, the BMS implementation of PCM [35]. They found an increase of $\eta / s$ for decreasing temperature.

From Eq. (12) the shear viscosity $\eta$ is proportional to the product of the energy density $e$ and the relaxation time $\tau$. It is clear that the relaxation time should be a product of the mean free path $\lambda_{m f p}$ and a function of the distribution of the collision angle $F[d(\theta)]$, i.e., 


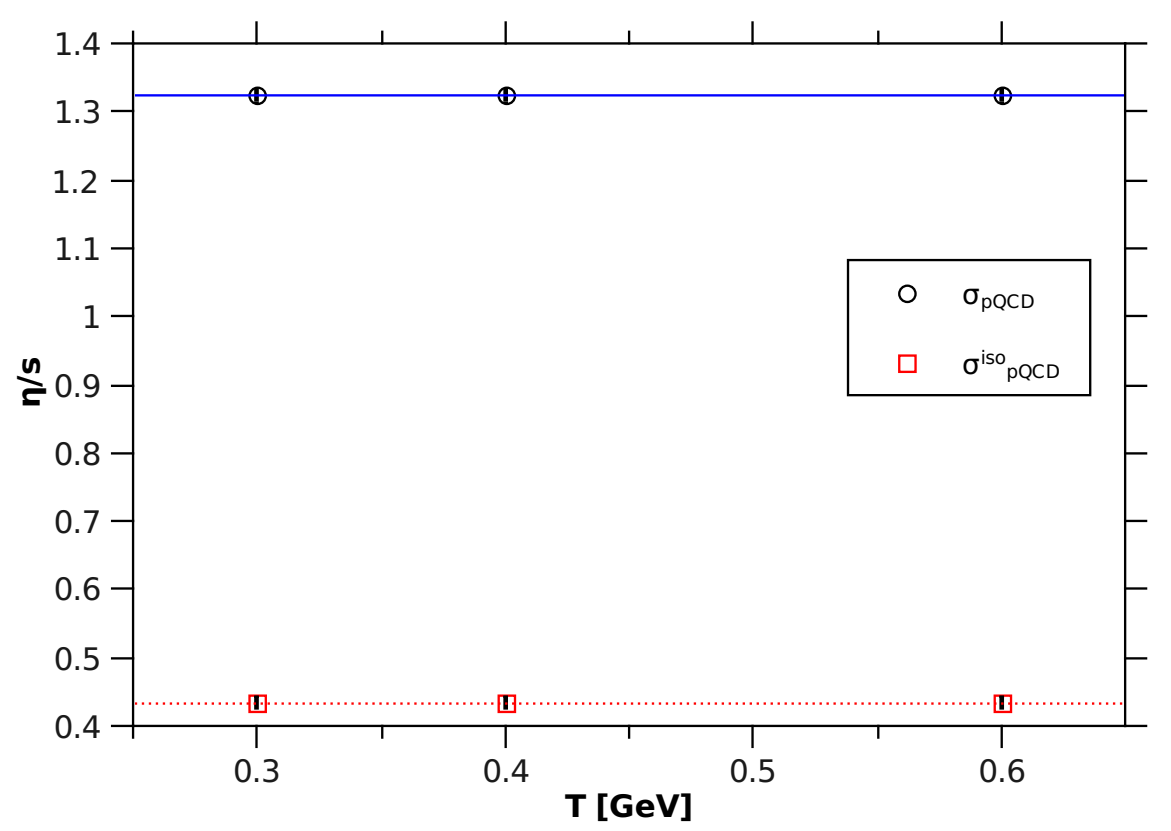

FIG. 5: (Color online) Temperature dependence of the $\eta / s$ ratio. PQCD (circles) and isotropic (squares) distribution of the collision angle in elastic gluon interactions are taken for comparisons.

$\tau \sim \lambda_{m f p} F[d(\theta)]$. From Eq. 15 we obtain

$$
\begin{aligned}
& \sigma_{g g \rightarrow g g}=\frac{9 \pi \alpha_{s}^{2}}{2} \frac{1}{m_{D}^{2}\left(1+4 m_{D}^{2} / \hat{s}\right)} \\
& d(\theta)=\frac{1}{\sigma_{g g \rightarrow g g}} \frac{d \sigma_{g g \rightarrow g g}}{d \theta}=\frac{4 \sin (2 \theta) m_{D}^{2}\left(\hat{s}+4 m_{D}^{2}\right)}{\left(\hat{s} \sin ^{2} \theta+4 m_{D}^{2}\right)^{2}} .
\end{aligned}
$$

Because momenta of gluons are distributed with $f(p) \sim e^{-E / T}$, it is obvious that $\hat{s}$ scales with $T^{2}, \sigma_{g g \rightarrow g g}$ with $1 / T^{2}$, and $d(\theta)$ has no dependence on $T$. The mean free path $\lambda_{m f p}=$ $1 / n\left\langle\sigma_{g g \rightarrow g g}\right\rangle$ is then proportional to $1 / T$, and $\eta \sim e \lambda_{m f p} F[d(\theta)]$ is proportional to $T^{3}$, like $s$. Thus, $\eta / s$ does not depend on the temperature for the case Eq. (15).

We now turn to discuss the dependence of the shear viscosity on the distribution of the collision angle. In analogy to the procedure in [25] we use the total cross section (18) to determine the collision probability (3) and then replace the angular distribution (19) by an isotropic form $d_{i s o}(\theta) \sim \sin \theta$. The results on $\eta / s$ are also shown in Fig. 5. The $\eta / s$ ratio is smaller by a factor of 3 if the pQCD angular distribution is replaced by an isotropic one.

To understand the dependence of the shear viscosity on the angular distribution, we calculate the transport cross section, $\sigma^{t r}=\int d \theta \sin ^{2} \theta d \sigma / d \theta$, which is roughly inversely 
proportional to $\eta$. For the isotropic angular distribution we have $\sigma^{t r}=(2 / 3) \sigma$, while for the pQCD angular distribution

$$
\frac{\sigma_{g g \rightarrow g g}^{t r}}{\sigma_{g g \rightarrow g g}}=a(1+a)[\ln (1+1 / a)-1 /(1+a)]
$$

with $a=4 m_{D}^{2} / \hat{s}$. On the average $\langle\hat{s}\rangle=18 T^{2}$ and thus $\langle a\rangle=16 \alpha_{s} /(3 \pi) \approx 0.5$ for $\alpha_{s}=0.3$.

We obtain $\sigma_{g g \rightarrow g g}^{t r}=0.32 \sigma_{g g \rightarrow g g}$, which is a factor of 2 smaller than that using the isotropic angular distribution. By this analytical estimation the shear viscosity effectively decreases by a factor of two if one replaces the forward-backward peaked pQCD angular distribution by an isotropic one. From numerical calculations we see a factor of 3 as seen in Fig. 5. This situation for elastic scatterings also indicates the efficiency of gluon bremsstrahlung $g g \leftrightarrow g g g$ in thermalization and lowering the shear viscosity if the fact of the finite radiation angle at moderate $\alpha_{s}$ is taken into account.

\section{SUMMARY}

In this work we calculated the shear viscosity with the Green-Kubo relation. The correlation function is extracted from simulations by the parton cascade BAMPS. To cross-check the numerical method, the shear viscosity for interactions with constant cross section and isotropic angular distribution is calculated and the results agree perfectly with the analytical ones. The main results are the values of $\eta / s$ ratio of a gluon gas including a $2 \leftrightarrow 3$ pQCDbased gluon bremsstrahlung process. These results are in perfect agreement with previously published numbers employing other methods. In comparison to pure elastic processes the the $\eta / s$ ratio is lowered by a factor of $3-8$ for $\alpha_{s}=0.01-0.6$ compared to solely elastic collisions when gluon bremsstrahlung is included. A nice scaling of $\eta \sim 1 /\left[\alpha_{s}^{2} \ln \left(1.5 / \alpha_{s}\right)\right]$ in the coupling constant $\alpha_{s}$ is found as expected from standard pQCD arguments. We also showed that $\eta / s$ has no temperature dependence at constant $\alpha_{s}$ and the change of the pQCD elastic interactions to those with isotropic angular distribution brings a factor of 3 decrease in the shear viscosity.

\section{ACKNOWLEDGEMENTS}

The authors are grateful to the Center for the Scientific Computing (CSC) at Frankfurt for the computing resources. C. Wesp, C.Greiner and Z. Xu thank S. Bass for fruitful 
discussions. C. Wesp, F. Reining, A. El and I. Bouras are grateful to HGS-HIRe. C. Wesp and A. El are grateful to H-QM. This work was supported by the Helmholtz International Center for FAIR within the framework of the LOEWE program launched by the State of Hessen.

[1] K. H. Ackermann et al., Phys. Rev. Lett. 86, 402 (2001).

[2] S. S. Adler et al. (PHENIX Collaboration), Phys. Rev. Lett. 91, 182301 (2003).

[3] J. Adams et al. (STAR Collaboration), Phys. Rev. Lett. 92, 052302 (2004).

[4] B. B. Back et al., Phys. Rev. Lett. 94, 122303 (2005).

[5] B. Alver et al., Phys. Rev. Lett. 98, 242302 (2007).

[6] S. Afanasiev et al. (PHENIX Collaboration), Phys. Rev. C 80, 024909 (2009).

[7] K. Aamodt et al. (ALICE Collaboration), (2010), arXiv:1011.3914 [nucl-ex].

[8] H. Song and U. W. Heinz, Phys.Rev. C77, 064901 (2008).

[9] M. Luzum and P. Romatschke, Phys. Rev. C 78, 034915 (2008).

[10] D. A. Teaney, (2009), arXiv:0905.2433 [nucl-th].

[11] B. Schenke, S. Jeon, and C. Gale, Phys.Rev. C82, 014903 (2010).

[12] H. Song, S. A. Bass, and U. W. Heinz, (2011), arXiv:1103.2380 [nucl-th]

[13] B. Schenke, S. Jeon, and C. Gale, (2011), arXiv:1102.0575 [hep-ph].

[14] Z. Xu and C. Greiner, Phys. Rev. C 71, 064901 (2005).

[15] Z. Xu., C. Greiner, and H. Stöcker, Phys. Rev. Lett. 101, 082302 (2008).

[16] G. Ferini, M. Colonna, M. Di Toro, and V. Greco, Phys. Lett. B670, 325 (2009).

[17] D. Molnar and M. Gyulassy, Nucl. Phys. A698, 379 (2002).

[18] P. Arnold, G. D. Moore, and L. G. Yaffe, Journal of High Energy Physics 2003, 051 (2003).

[19] P. Kovtun, D. Son, and A. Starinets, Phys.Rev.Lett. 94, 111601 (2005).

[20] M. Bluhm, B. Kampfer, and K. Redlich, arXiv:1011.5634 [hep-ph] (2010).

[21] Z. Xu and C. Greiner, Phys. Rev. Lett. 100, 172301 (2008).

[22] A. El, A. Muronga, Z. Xu, and C. Greiner, Phys. Rev. C79, 044914 (2009).

[23] J.-W. Chen, H. Dong, K. Ohnishi, and Q. Wang, Phys. Lett. B685, 277 (2010).

[24] J.-W. Chen, J. Deng, H. Dong, and Q. Wang, Phys. Rev. D83, 034031 (2011).

[25] J. Fuini, III, N. S. Demir, D. K. Srivastava, and S. A. Bass, J. Phys. G38, 015004 (2011). 
[26] M. S. Green, Journal of Chemical Physics 22, 398 (1954).

[27] R. Kubo, Journal of the Physical Society of Japan 12, 570 (1957).

[28] L. Onsager, PhysRev.37.405-426n 37, 405 (1931).

[29] R. Zubarev, Morozov, Statistical Mechanics of Nonequilibrium Processes Volume 2: Relaxation and Hydrodynamic Processes (Akademie Verlag GmbH, 1996).

[30] R. Zubarev, Morozov, Statistical Mechanics of Nonequilibrium Processes Volume 1: Basic Concepts, Kinetic Theory (Akademie Verlag GmbH, 1996).

[31] E. A. Calzetta, Nonequilibrium Quantum Field Theory (Cambridge University Press, 2008).

[32] D. J. Searles and D. J. Evans, The Journal of Chemical Physics 112, 9727 (2000).

[33] S. A. Bass et al., Prog. Part. Nucl. Phys. 41, 255 (1998).

[34] M. Bleicher et al., J. Phys. G25, 1859 (1999).

[35] S. A. Bass, B. Muller, and D. K. Srivastava, Phys. Lett. B551, 277 (2003).

[36] N. Demir and S. A. Bass, Phys. Rev. Lett. 102, 172302 (2009).

[37] Z. Xu and C. Greiner, Phys. Rev. C 76, 024911 (2007).

[38] C. Wesp, Shear viscosity, Green-Kubo relations and transport simulations - unpublished, Master's thesis, Goethe-Universität Frankfurt am Main (2010).

[39] L. E. Reichl, A Modern Course in Statistical Mechanics (Hodder \& Stoughton Educational, 1980).

[40] T. Hirano and M. Gyulassy, Nucl.Phys. A769, 71 (2006).

[41] S. de Groot and W. van Leeuwen, Relativistic kinetic theory : principles and applications / (North-Holland, 1980).

[42] P. Huovinen and D. Molnar, Phys.Rev. C79, 014906 (2009).

[43] A. El, A. Muronga, Z. Xu, and C. Greiner, Nucl. Phys. A848, 428 (2010).

[44] A. El, F. Lauciello, et al., Paper in preparation.

[45] J. Gunion and G. Bertsch, Phys.Rev. D25, 746 (1982).

[46] A. B. Migdal, Phys. Rev. 103, 1811 (1956).

[47] J.-P. Blaizot, E. Iancu, and A. Rebhan, Phys. Rev. Lett. 83, 2906 (1999).

[48] G. Baym, H. Monien, C. J. Pethick, and D. G. Ravenhall, Phys. Rev. Lett. 64, 1867 (1990).

[49] P. B. Arnold, G. D. Moore, and L. G. Yaffe, JHEP 11, 001 (2000).

[50] A. Peshier and W. Cassing, Phys. Rev. Lett. 94, 172301 (2005).

[51] A. Khvorostukhin, V. Toneev, and D. Voskresensky, (2010), arXiv:1011.0839 [nucl-th]. 
[52] S. Plumari, W. M. Alberico, V. Greco, and C. Ratti, (2011), arXiv:1103.5611 [hep-ph],

[53] O. Fochler, Z. Xu, and C. Greiner, Phys.Rev. C82, 024907 (2010).

[54] F. Reining et al., "Extraction of shear viscosity in stationary states of relativistic particle systems," Paper in preperation. 